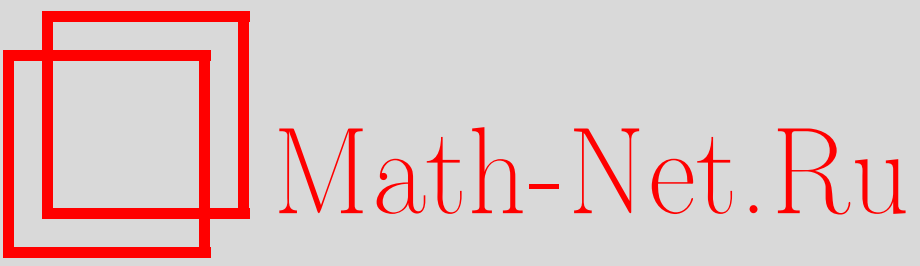

М. Маньяс, Л. Мартинес Алонсо, Е. Медина, Редукции бездисперсионной иерархии КП, ТМФ, 2002, том 133, номер 3, 463-474

DOI: https://doi.org/10.4213/tmf411

Использование Общероссийского математического портала Math-Net.Ru подразумевает, что вы прочитали и согласны с пользовательским соглашением

http://www.mathnet.ru/rus/agreement

Параметры загрузки:

IP : 3.81 .55 .215

26 апреля 2023 г., 14:46:01

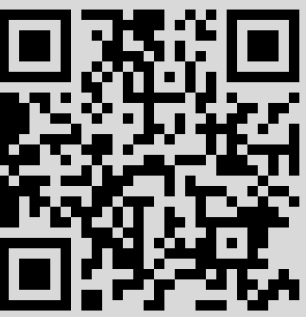


ТЕОРЕТИЧЕСКАЯ

И МАТЕМАТИЧЕСКАЯ

ФИЗИКА

Том 133, № 3

декабрь, 2002

(C) 2002 г. М. Маньяс*, Л. Мартинес Алонсо*, Е. Медина ${ }^{\dagger}$

\section{РЕДУКЦИИ БЕЗДИСПЕРСИОННОЙ ИЕРАРХИИ КП}

Предложен метод построения $S$-функции, основанный на системе дифференциальных уравнений первого порядка. Этот метод применен для анализа редукций бездисперсионных интегрируемых иерархий.

Ключевые слова: преобразования типа годограффа, редукции бездисперсионных иерархий.

\section{1. ВВЕДЕНИЕ}

В течение последнего десятилетия бездисперсионные интегрируемые системы привлекают к себе все бо́льший интерес. Этот возрастающий интерес обусловлен приложениями этих систем к проблеме классификации в топологической полевой теории [1], исследованию систем гидродинамического типа [2] и теории конформных отображений [3]. Редукции этих систем рассматривались в работах [2], [4].

В данной работе разрабатывается общая схема исследования редукций бездисперсионных интегрируемых систем. В основе этой схемы лежит метод нахож дения $S$-функции, введенный в работе [5]. Это позволяет находить как редукции, так и решения типа годографа. Указанная схема применяется к исследованию бездисперсионной иерархии Кадомцева-Петвиашвили (КП). Также исследуются различные характерные примеры, для которых найдены обширные семейства решений некоторых редукций бездисперсионной иерархии КП.

\section{2. РЕДУКЦИИ БЕЗДИСПЕРСИОННОЙ ИЕРАРХИИ КП}

2.1. Характерные свойства редукции. Для того чтобы ввести бездисперсионную иерархию КП, рассмотрим функцию $z=z(p, \mathbf{t})$, зависящую от комплексной переменной $p$ и от бесконечного набора комплексных параметров типа времен $\mathbf{t}:=(x:=$

*Departamento de Física Teórica II, Universidad Complutense, Madrid, Spain. E-mail: manuel@arboux.fis.ucm.es, luism@eucmos.sim.ucm.es

${ }^{\dagger}$ Departamento de Matemáticas, Universidad de Cádiz, Puerto Real, Cádiz, Spain. E-mail: elena.medina@uca.es 
$\left.t_{1}, t_{2}, \ldots\right)$, которая допускает разложение в ряд вида

$$
z=p+\sum_{n=1}^{\infty} \frac{a_{n}(\mathbf{t})}{p^{n}}, \quad p \rightarrow \infty
$$

Тогда бездисперсионная иерархия КП [4]-[6] представляет собой набор уравнений

$$
\frac{\partial z}{\partial t_{n}}=\left\{\Omega_{n}, z\right\}, \quad \Omega_{n}:=\left(z^{n}\right)_{+}, \quad n \geqslant 1
$$

где через $\{\cdot, \cdot$ · $\}$ обозначена скобка Пуассона, определенная по отношению к переменным $p$ и $x$, а $\Omega_{n}=\left(z^{n}\right)+$ обозначает полиномиальную часть $z^{n}$ как функции от $p$. Например,

$$
(z)_{+}=p, \quad\left(z^{2}\right)_{+}=p^{2}+2 a_{1}, \quad\left(z^{3}\right)_{+}=p^{3}+3 p a_{1}+3 a_{2} .
$$

Условия совместности системы (2)

$$
\frac{\partial \Omega_{m}}{\partial t_{n}}-\frac{\partial \Omega_{n}}{\partial t_{m}}+\left\{\Omega_{m}, \Omega_{n}\right\}=0, \quad m \neq n,
$$

образуют иерархию нелинейных дифференциальных уравнений в частных производных. Например, при $m=3, n=2$ получается бездисперсионное уравнение КП

$$
\left(u_{t_{3}}+3 u u_{x}\right)_{x}=\frac{3}{4} u_{t_{2} t_{2}}, \quad u:=-a_{1}, \quad y:=t_{2}, \quad t:=t_{3}
$$

Известно [5], что сушествует $S$-функиия $S=S(z, \mathbf{t})$ такая, что

$$
\begin{gathered}
\frac{\partial S(z)}{\partial t_{n}}=\Omega_{n}(p, \mathbf{t}), \quad n \geqslant 1, \\
S(z, \mathbf{t})=\sum_{n \geqslant 1} z^{n} t_{n}+\sum_{n \geqslant 1} \frac{S_{n}(\mathbf{t})}{z^{n}}, \quad z \rightarrow \infty .
\end{gathered}
$$

Полагая $n=1$ в (4), получим

$$
p=z+\sum_{n \geqslant 1} \frac{b_{n}(\mathbf{t})}{z^{n}}, \quad b_{n}:=\frac{\partial S_{n}}{\partial x}
$$

и тогда можно доказать (см. [5]), что обратный ряд задает решение $z=z(p, \mathbf{t})$ бездисперсионной иерархии КП. Условия (4), определяющие $S$-функцию, образуют систему совместных уравнений типа Гамильтона-Якоби, которая представляет собой квазиклассический предел системы линейных уравнений для волновой функции стандартной иерархии КП. 
Из условий (5) и (6) следует, что функция $S$, допускающая разложение (5), удовлетворяет системе (4) тогда и только тогда, когда

$$
\left(\frac{\partial S(z)}{\partial t_{n}}\right)_{-}=0, \quad n \geqslant 1
$$

Здесь и ниже будем рассматривать $S$ как функцию, зависящую от $z$ или от $p$, и будем обозначать соответствуюшие зависимости как $S(z)$ и $S(p)(S(z, \mathbf{t})=S(p(z, \mathbf{t}), \mathbf{t}))$. Обозначим через $S_{+}(p)$ и $S_{-}(p)$ части в разложении $S(p)$, содержашие соответственно положительные и отрищательные степени $p, S(p)=S_{+}(p)+S_{-}(p)$. Из условий (5) и (6) получим, что

$$
S_{+}(p)=\sum_{n \geqslant 1} \Omega_{n}(p, \mathbf{t}) t_{n} .
$$

Тогда уравнение (7) приводит к условию

$$
\left(\frac{\partial S(p)}{\partial p} \frac{\partial p}{\partial t_{n}}+\frac{\partial S_{-}(p)}{\partial t_{n}}\right)_{-}=0, \quad n \geqslant 1
$$

Рассмотрим теперь $N$-редукции бездисперсионной иерархии КП, для которых $z=$ $z(p, \mathbf{t})$ зависит от $\mathbf{t}$ только через $N$ функций $\mathbf{u}=\left(u_{1}(\mathbf{t}), \ldots, u_{N}(\mathbf{t})\right)$. Будем характеризовать эти редукции с помошью систем уравнений на $p=p(z, \mathbf{u})$, имеющих вид

$$
\frac{\partial p}{\partial u_{i}}=R_{i}(p, \mathbf{u}), \quad i=1, \ldots, N
$$

или, эквивалентно, с помощью систем уравнений на $z=z(p, \mathbf{u})$

$$
\frac{\partial z}{\partial u_{i}}+R_{i}(p, \mathbf{u}) \frac{\partial z}{\partial p}=0, \quad i=1, \ldots, N
$$

Будем предполагать, что функции $R_{i}$ удовлетворяют следующим условиям.

1. Функции $R_{i}$ суть рациональные функции от $p$, сингулярности которых - это $N$ простых полюсов $p_{i}=p_{i}(\mathbf{u}), i=1, \ldots, N$, и которые обрашаются в нуль при $p=\infty$. Тем самым эти функции допускают разложение

$$
R_{i}(p, \mathbf{u})=\sum_{j=1}^{N} \frac{r_{i j}(\mathbf{u})}{p-p_{j}(\mathbf{u})}
$$

2. Функции $R_{i}$ удовлетворяют условиям совместности системы (11):

$$
\frac{\partial R_{i}}{\partial u_{j}}-\frac{\partial R_{j}}{\partial u_{i}}+R_{j} \frac{\partial R_{i}}{\partial p}-R_{i} \frac{\partial R_{j}}{\partial p}=0, \quad i \neq j
$$

5 Теоретическая и математическая физика, т. 133, № 3, 2002 г. 
Также предполагается, что $S_{-}(p)$ зависит от $\mathbf{t}$ только через функции $\mathbf{u}=\mathbf{u}(\mathbf{t})$. Тогда условия (9) принимают вид

$$
\left(\frac{\partial S(p)}{\partial p} R_{i}+\frac{\partial S_{-}(p)}{\partial u_{i}}\right)_{-}=0
$$

Чтобы построить решения уравнений редукции, будем предполагать, что функция $S$ удовлетворяет условиям

$$
\frac{\partial S}{\partial p}\left(p_{i}\right)=0, \quad i=1, \ldots, N .
$$

Пусть теперь $E=E(p, \mathbf{u})$ обозначает любую целую функцию от $p$, удовлетворяющую уравнениям

$$
E\left(p_{i}, \mathbf{u}\right)=F_{i}(\mathbf{u}), \quad i=1, \ldots, N,
$$

где

$$
F_{i}(\mathbf{u}):=\frac{\partial S_{-}}{\partial p}\left(p_{i}\right)
$$

В терминах этой функции получим, что условия (14) эквивалентны условиям

$$
\frac{\partial S_{-}(p)}{\partial u_{i}}+R_{i} \frac{\partial S_{-}(p)}{\partial p}=\left(E R_{i}\right)_{-} .
$$

Далее, используя (13), получим, что условия совместности системы (17) имеют вид

$$
\frac{\partial\left(E R_{i}\right)_{-}}{\partial u_{j}}-\frac{\partial\left(E R_{j}\right)_{-}}{\partial u_{i}}+R_{j} \frac{\partial\left(E R_{i}\right)_{-}}{\partial p}-R_{i} \frac{\partial\left(E R_{j}\right)_{-}}{\partial p}=0, \quad i \neq j .
$$

Поскольку справедливо разложение

$$
\left(E R_{j}\right)_{-}=\sum_{k=1}^{N} \frac{r_{j k} F_{k}}{p-p_{k}}
$$

получим, что уравнения (18) образуют набор условий согласования для функций $F_{j}$.

Подводя итог, в случае, когда рассмотрение начинается с набора функций $R_{i}(p, \mathbf{u})$ и $F_{i}(\mathbf{u})(i=1, \ldots, N)$, удовлетворяющих условиям (13) и (18), можно получить решение $N$-редукции бездисперсионной иерархии КП из уравнений (11). Кроме того, из условий (15) и (16) имеем

$$
\frac{\partial S_{+}}{\partial p}\left(p_{i}\right)+F_{i}(\mathbf{u})=0
$$

или, эквивалентно,

$$
\sum_{n=1}^{\infty} v_{i n}(\mathbf{u}) t_{n}+F_{i}(\mathbf{u})=0, \quad v_{i n}:=\frac{\partial \Omega_{n}}{\partial p}\left(p_{i}\right), \quad i=1, \ldots, N .
$$

Эта система порождает неявные соотношения на функции $\mathbf{u}=\mathbf{u}(\mathbf{t})$, которые характеризуют $N$-редукции. Наконец, из условий (5) и (17) следует, что величины $S$ - могут быть получены рекурсивно. Ввиду формы записи неявных соотношений (20) естественно называть такие решения решениями типа годографа. 
2.2. Интегрируемость по Бурле. В работах [7] $S$-функции были построены с помощью метода обратной задачи, что позволило решить задачу на начальные данные для нескольких бездисперсионных моделей. Анализ в данной работе базируется на альтернативном методе нахождения функции $S$, основанном на системе дифференциальных уравнений (10) и (17). $S$-функция при этом задается набором спектральных данных $\left\{p_{i}(\mathbf{u}), r_{i j}(\mathbf{u}), F_{i}(\mathbf{u}): 1 \leqslant i, j \leqslant N\right\}$. Кроме того, из (12) и (19) следует, что условия совместности (13) и (18) эквивалентны следуюшим условиям совместности для спектральных данных:

$$
\begin{aligned}
r_{i k} \frac{\partial p_{k}}{\partial u_{j}}-r_{j k} \frac{\partial p_{k}}{\partial u_{i}} & =\sum_{l \neq k} \frac{r_{j l} r_{i k}-r_{i l} r_{j k}}{p_{k}-p_{l}}, \\
\frac{\partial r_{i k}}{\partial u_{j}}-\frac{\partial r_{j k}}{\partial u_{i}} & =2 \sum_{l \neq k} \frac{r_{j k} r_{i l}-r_{i k} r_{j l}}{\left(p_{k}-p_{l}\right)^{2}}, \\
r_{i k} \frac{\partial F_{k}}{\partial u_{j}}-r_{j k} \frac{\partial F_{k}}{\partial u_{i}} & =\sum_{l \neq k} \frac{r_{j l} r_{i k}-r_{i l} r_{j k}}{\left(p_{k}-p_{l}\right)^{2}}\left(F_{k}-F_{l}\right),
\end{aligned}
$$

где $i \neq j$. Две первые группы уравнений системы (21) задают редукции бездисперсионной иерархии КП, в то время как вся система определяет набор решений типа годографа.

Можно показать, что система (21) согласована в том смысле, что условия

$$
\begin{gathered}
\frac{\partial}{\partial u_{m}} \frac{\partial p_{k}}{\partial u_{l}}=\frac{\partial}{\partial u_{l}} \frac{\partial p_{k}}{\partial u_{m}} \\
\frac{\partial}{\partial u_{m}} \frac{\partial r_{i k}}{\partial u_{l}}=\frac{\partial}{\partial u_{l}} \frac{\partial r_{i k}}{\partial u_{m}} \\
\frac{\partial}{\partial u_{m}} \frac{\partial F_{k}}{\partial u_{l}}=\frac{\partial}{\partial u_{l}} \frac{\partial F_{k}}{\partial u_{m}}
\end{gathered}
$$

выполнены, если выполняются уравнения (21). Можно также увидеть, что система уравнений (21) эквивалентна следующей системе:

$$
\begin{aligned}
\frac{\partial p_{k}}{\partial u_{i}} & =\frac{1}{r_{s_{k} k}}\left(r_{i k} \frac{\partial p_{k}}{\partial u_{s_{k}}}-\sum_{l \neq k} \frac{r_{s_{k} l} r_{i k}-r_{i l} r_{s_{k} k}}{p_{k}-p_{l}}\right), \quad i<s_{k} \\
\frac{\partial p_{k}}{\partial u_{i}} & =-\frac{1}{r_{s_{k} k}} \sum_{l \neq k} \frac{r_{s_{k} l} r_{i k}-r_{i l} r_{s_{k} k}}{p_{k}-p_{l}}, \quad i>s_{k} \\
\frac{\partial F_{k}}{\partial u_{i}} & =\frac{1}{r_{s_{k} k}}\left(r_{i k} \frac{\partial F_{k}}{\partial u_{s_{k}}}-\sum_{l \neq k} \frac{r_{s_{k} l} r_{i k}-r_{i l} r_{s_{k} k}}{\left(p_{k}-p_{l}\right)^{2}}\left(F_{k}-F_{l}\right)\right), \quad i<s_{k} \\
\frac{\partial F_{k}}{\partial u_{i}} & =-\frac{1}{r_{s_{k} k}} \sum_{l \neq k} \frac{r_{s_{k} l} r_{i k}-r_{i l} r_{s_{k} k}}{\left(p_{k}-p_{l}\right)^{2}}\left(F_{k}-F_{l}\right), \quad i>s_{k}, \\
\frac{\partial r_{i k}}{\partial u_{j}} & =\frac{\partial r_{j k}}{\partial u_{i}}+2 \sum_{l \neq k} \frac{r_{j k} r_{i l}-r_{i k} r_{j l}}{\left(p_{k}-p_{l}\right)^{2}}, \quad i>j
\end{aligned}
$$


для $k=1, \ldots, N$, где для каждого $k$ должно вьполняться условие, что число $s_{k} \in$ $\{1, \ldots, N\}$ таково, что $r_{s_{k}} \neq 0$ и $r_{i k}=0$ при $i>s_{k}$. Система уравнений (22) имеет вид системы Бурле [8]. Чтобы это показать, заметим, что $\left(u_{1}, \ldots, u_{s_{k}-1}, u_{s_{k}+1}, u_{N-1}\right)$ являются главными переменными для $p_{k}$ и $F_{k}$, в то время как переменные $u_{s_{k}}$ оказываются параметрическими. Аналогично $\left(u_{1}, \ldots, u_{i-1}\right)$ суть главные переменные для $r_{i k}$, в то время как переменные $\left(u_{i}, \ldots, u_{N}\right)$ оказываются параметрическими. Условия совместности по главным переменным могут быть проверены непосредственно. С другой стороны, поскольку $r_{k s_{k}} \neq 0$ и $p_{k} \neq p_{l}, k, l=1, \ldots, N, k \neq l$, получим, что функции, задаюшие систему, являются аналитическими. Поэтому, применив теорему Бурле, можно заключить, что в окрестности исходной точки $\mathbf{u}_{0}=\left(u_{1}^{(0)}, \ldots, u_{N}^{(0)}\right)$ сушествует единственное решение $\left\{p_{k}, F_{k}, r_{i k}\right\}$ такое, что в момент, когда главные переменные принимают исходные значения, решение представляет собой набор произвольных аналитических функций от соответствуюших параметрических переменных. Обшее решение тем самым зависит от $N(N+1)$ произвольных аналитических функций параметрических переменных: $3 N$ функций одной переменной и $N$ аналитических функций от $l$ переменных для каждого $l=2, \ldots, N-1$.

2.3. Системы гидродинамического типа. Неявные уравнения (20) представляют собой преобразования типа годографа. Это предполагает наличие уравнений гидродинамического типа. Действительно, предполагая, что $z=z(p, \mathbf{u})$ есть регулярная функция вблизи точек $p_{i}$, из условий (11) можно найти, что

$$
\frac{\partial z}{\partial p}\left(p_{i}\right)=0, \quad i=1, \ldots, N
$$

Используя теперь уравнения (2), получим

$$
\sum_{j=1}^{N} \frac{\partial z_{i}}{\partial u_{j}} \frac{\partial u_{j}}{\partial t_{n}}=v_{i n} \sum_{j=1}^{N} \frac{\partial z_{i}}{\partial u_{j}} \frac{\partial u_{j}}{\partial x}, \quad n \geqslant 1
$$

где $z_{i}:=z\left(p_{i}, \mathbf{u}(\mathbf{t})\right)$. Таким образом, выражая $\mathbf{u}(\mathbf{t})$ через функции $z_{i}$, найдем, что вектор-функция $\mathbf{u}(\mathbf{t})$ удовлетворяет системе уравнений гидродинамического типа:

$$
\begin{gathered}
\frac{\partial \mathbf{u}}{\partial t_{n}}=A_{n}(\mathbf{u}) \frac{\partial \mathbf{u}}{\partial x}, \quad n=1, \ldots, N \\
\mathbf{u}=\left(\begin{array}{c}
u_{1} \\
\vdots \\
u_{N}
\end{array}\right), \quad A_{n}:=K^{-1} D_{n} K, \\
D_{n}:=\operatorname{diag}\left(v_{1 n}, \ldots, v_{N n}\right), \quad K_{i j}:=\frac{\partial z_{i}}{\partial u_{j}} .
\end{gathered}
$$


Принимая во внимание, что $v_{2 i}=2 p_{i}$, из системы (23) можно получить формулу Гиббонса-Кодамы [2]

$$
A_{n}=v_{n}(A), \quad A:=\frac{A_{2}}{2},
$$

где $v_{n}(p):=\partial \Omega_{n} / \partial p$. Наконец, если предположить, что функции $\partial_{x} u_{j}, j=1, \ldots, N$, независимы, можно выразить рациональные функции $R_{i}$ в терминах матрицы Гиббонса-Кодамы $A$ в виде

$$
R_{i}(p, \mathbf{u})=\sum_{j=1}^{N}(A(\mathbf{u})-p)_{j i}^{-1} \frac{\partial a_{1}}{\partial u_{j}} .
$$

Из представления (25) следует, что

$$
r_{i k}=-\frac{\partial z_{k}}{\partial u_{i}} r_{k}, \quad r_{k}:=\frac{\partial a_{1}}{\partial z_{k}} .
$$

В новых координатах $\left\{z_{i}\right\}_{i=1}^{N}$ система (21) принимает вид

$$
\begin{aligned}
\frac{\partial r_{i}}{\partial z_{j}} & =2 \frac{r_{i} r_{j}}{\left(p_{j}-p_{i}\right)^{2}}, \\
\frac{\partial p_{i}}{\partial z_{j}} & =\frac{r_{j}}{p_{j}-p_{i}}, \\
\frac{\partial F_{i}}{\partial z_{j}} & =r_{j} \frac{F_{j}-F_{i}}{\left(p_{j}-p_{i}\right)^{2}} .
\end{aligned}
$$

Заметим, что в соответствии с этой системой получим, что

$$
\frac{\partial F_{i}}{\partial z_{j}} \frac{1}{F_{j}-F_{i}}=\frac{\partial p_{i}}{\partial z_{j}} \frac{1}{p_{j}-p_{i}}=\frac{1}{2} \frac{\partial \ln r_{i}}{\partial z_{j}}, \quad i \neq j .
$$

Эти соотношения задают геометрическую интерпреташию системы (21). Вводя новые величины

$$
\beta_{i j}:=\frac{1}{\sqrt{r_{i}}} \frac{\partial \sqrt{r_{j}}}{\partial z_{i}}=\frac{\sqrt{r_{i} r_{j}}}{\left(p_{i}-p_{j}\right)^{2}}=\beta_{j i}, \quad i \neq j,
$$

получим семейство параллельных сопряженных сетей $\mathbf{x}=\mathbf{x}(\mathbf{u})$, задаваемых решениями системы

$$
\frac{\partial \mathbf{x}}{\partial z_{i}}=H_{i} \mathbf{X}_{i}
$$

где $H_{i}$ и $\mathbf{X}_{i}$ (соответственно коэффициенты Ламе и перенормированные касательные векторы) задаются уравнениями

$$
\frac{\partial H_{i}}{\partial z_{i}}=\beta_{j i} H_{j}
$$

и

$$
\frac{\partial \mathbf{X}_{i}}{\partial z_{i}}=\beta_{i j} \mathbf{X}_{j}
$$

Очевидно, что коэффициенты Ламе $H_{i}:=\sqrt{r_{i}}$ дают решение системы $(29)$, и тогда из системы уравнений (26) следует, что $F_{i} H_{i}$ и $p_{i} H_{i}$ также представляют собой решения системы (29). 


\section{3. ПРИМЕРЫ}

3.1. $(N=1)$-редукции. Если в редукции участвует только одна функция $u=u(\mathbf{t})$, то полагая $u=-a_{1}$, можно свести уравнение (10) к уравнению Абеля

$$
\frac{\partial p}{\partial u}=\frac{1}{p-p_{1}(u)}
$$

где $p_{1}$ - произвольная функция (набор условий совместности в этом случае, очевидно, пуст). Из системы (11) при этом следует рекуррентное соотношение на коэффициенты разложения функции $z=z(p, u)$ :

$$
\begin{gathered}
a_{1}=-u, \quad a_{2}=-\int p_{1}(u) d u, \\
a_{m+2}^{\prime}=p_{1}(u) a_{m+1}^{\prime}+m a_{m}, \quad m \geqslant 1,
\end{gathered}
$$

где $a_{m}^{\prime}:=\partial a_{m} / \partial u$. Теперь можно использовать это разложение и формулу (20), для того чтобы порождать решения уравнений бездисперсионной иерархии КП. Например, полагая $t_{n}=0, n \geqslant 4$, можно свести (20) к уравнению Кодамы [4] для $(N=1)$-редукции бездисперсионного уравнения КП.

Явное выражение для решения $z=z(p, u)$ системы (11) удается получить лишь в некоторых частных случаях. Например, простейший случай отвечает условию $p_{1}(u) \equiv 0$ (бездисперсионная редукция уравнения Кортевега-де Фриза). При этом получим

$$
z=\left(p^{2}-2 u\right)^{1 / 2} .
$$

С другой стороны, в случае, когда $p_{1} \equiv 0$, можно решить систему (17), задаюшую величину $S_{-}$, которая при этом оказьвается равна

$$
S_{-}(p, u)=-\left(\int_{0}^{z(p, u)} F\left(\frac{1}{2}\left(q^{2}-z(p, u)^{2}\right)\right) d q\right)_{-} .
$$

3.2. $(N=2)$-редукции. Рассмотрим теперь случай, когда $\mathbf{u}=(u, v)$ с $u=-a_{1}$. Из системы (25) при этом получим

$$
\begin{aligned}
& \frac{\partial p}{\partial u}=\frac{p-A_{22}}{\left(p-A_{11}\right)\left(p-A_{22}\right)-A_{12} A_{21}}, \\
& \frac{\partial p}{\partial v}=\frac{A_{12}}{\left(p-A_{11}\right)\left(p-A_{22}\right)-A_{12} A_{21}},
\end{aligned}
$$


где $A:=\left(A_{i j}(\mathbf{u})\right)-(2 \times 2)$-матрично-значная функция Гиббонса и Кодамы [2]. Правые части уравнений системы (32) имеют простые полюсы в точках

$$
A_{ \pm}:=\frac{1}{2}\left(\operatorname{tr} A \pm \sqrt{(\operatorname{tr} A)^{2}-4 \operatorname{det} A}\right)
$$

В этом случае уравнение (13) задает условия

$$
\partial_{v} A_{11}=\partial_{u} A_{12}, \quad\left(\begin{array}{c}
\partial_{v} \operatorname{det} A \\
-\partial_{u}(u+\operatorname{det} A)
\end{array}\right)=A\left(\begin{array}{c}
\partial_{v} \operatorname{tr} A \\
-\partial_{u} \operatorname{tr} A
\end{array}\right)
$$

Коэффициенты в разложении $z(p, \mathbf{u})$ определяются с помошью рекуррентных соотношений:

$$
\begin{gathered}
a_{1}=-u, \quad a_{2}=-\int A_{11} d u+A_{12} d v, \\
a_{3}=\int\left(\operatorname{det} A-u-A_{11} \operatorname{tr} A\right) d u-A_{12} \operatorname{tr} A d v, \\
\partial_{u} a_{m+2}=\operatorname{tr} A \partial_{u} a_{m+1}-\operatorname{det} A \partial_{u} a_{m}+m a_{m}-(m-1) A_{22} a_{m-1}, \\
\partial_{v} a_{m+2}=\operatorname{tr} A \partial_{v} a_{m+1}-\operatorname{det} A \partial_{v} a_{m}+(m-1) A_{12} a_{m-1} .
\end{gathered}
$$

Выбирая функцию $E$ в виде

$$
E:=p \frac{F_{+}-F_{-}}{A_{+}-A_{-}}+\frac{A_{+} F_{-}-A_{-} F_{+}}{A_{+}-A_{-}}
$$

где

$$
F_{ \pm}(\mathbf{u}):=\left.\frac{\partial S_{-}(p)}{\partial p}\right|_{A_{ \pm}}, \quad F:=\frac{A_{-} F_{+}-A_{+} F_{-}}{A_{+}-A_{-}}, \quad G:=\frac{F_{-}-F_{+}}{A_{+}-A_{-}}
$$

можно привести систему (18) к виду

$$
\left(\begin{array}{c}
-\partial_{v} F \\
\partial_{u} F
\end{array}\right)=A\left(\begin{array}{c}
\partial_{v} G \\
-\partial_{u} G
\end{array}\right)
$$

Таким образом, если $A$ и $F_{ \pm}$удовлетворяют соответствуюшим условиям совместности и накладываются условия $t_{n}=0$ при $n>4$, то первые потоки бездисперсионной иерархии КП могут быть найдены из решений соответствуюшей системы уравнений на $\mathbf{u}$ :

$$
4\left(A_{ \pm}^{3}-2 u A_{ \pm}-\int A_{11} d u+A_{12} d v\right) t_{4}+3\left(A_{ \pm}^{2}-u\right) t_{3}+2 A_{ \pm} t_{2}+x=-F_{ \pm}
$$


Если $t_{4}=0$, то эти уравнения становятся эквивалентными системе Кодамы для $(N=$ 2)-редукции [4]:

$$
\begin{gathered}
-3(u+\operatorname{det} A) t_{3}+x=F, \\
3 \operatorname{tr} A t_{3}+2 t_{2}=G .
\end{gathered}
$$

Особенно интересен случай, когда накладываются условия $u=-a_{1}, v=-a_{2}$, что отвечает выбору

$$
A=\left(\begin{array}{cc}
0 & 1 \\
-V & W
\end{array}\right), \quad V:=A_{+} A_{-}, \quad W:=A_{+}+A_{-} .
$$

При этом условия (33) принимают вид

$$
\begin{gathered}
\partial_{v} V+\partial_{u} W=0 \\
\partial_{u} V-V \partial_{v} W+W \partial_{v} V+1=0 .
\end{gathered}
$$

Поэтому, полагая

$$
V=\partial_{u} Z, \quad W=-\partial_{v} Z
$$

можно переформулировать (37) как уравнение Монжа-Ампера:

$$
\partial_{u u} Z+\partial_{u} Z \partial_{v v} Z-\partial_{v} Z \partial_{u v} Z+1=0
$$

Аналогично система (34) может быть представлена в виде

$$
\begin{gathered}
F=\partial_{u} T, \quad G=\partial_{v} T, \\
\partial_{u u} T+V \partial_{v v} T+W \partial_{u v} T=0 .
\end{gathered}
$$

Построим теперь некоторые решения бездисперсионного уравнения КП. Решение систем (37) и (39) имеет вид

$$
W=\frac{2 v}{u}, \quad V=\frac{v^{2}}{u^{2}}+c u^{2}+u, \quad T=k_{1} u+k_{2} v
$$

Решения типа годографа системы (3), отвечаюшие случаям $c \neq 0$ и $c=0$, суть соответственно

$$
u(x, y, t)=\frac{1}{6 c t}\left(-6 t+\sqrt{36 t^{2}+c\left[12 t\left(x-k_{1}\right)-\left(2 y-k_{2}\right)^{2}\right]}\right)
$$

и

$$
u(x, y, t)=\frac{12 t\left(x-k_{1}\right)-\left(2 y-k_{2}\right)^{2}}{72 t^{2}}
$$


Другое решение систем (37) и (39) имеет вид

$$
W=\frac{2 v}{u}, \quad V=\frac{v^{2}}{u^{2}}+u, \quad T=k \frac{v}{u}
$$

Оно приводит к решению типа годографа системы (3), неявно задаваемому алгебраическим уравнением

$$
72 t^{2} u^{3}+4\left(y^{2}-3 t x\right) u^{2}=k^{2}
$$

Можно также получить решение системы (3), неявно заданное трансцендентным уравнением, если выбрать

$$
W=c v+d, \quad V=a e^{c u}+\frac{1}{c}, \quad T=k_{1} u+k_{2} v, \quad a, c \neq 0 .
$$

В этом случае функция $u$ определяется из уравнения

$$
-3\left(u+a e^{c u}+\frac{1}{c}\right) t+\left(x-k_{1}\right)=0 .
$$

3.3. $(N=3)$-редукции. В этом случае предполагается, что функции $\mathbf{u}=(u, v, w)$ задаются первыми коэффициентами разложения $p=p(z, \mathbf{u})$,

$$
p=z+\frac{u}{z}+\frac{v}{z^{2}}+\frac{w}{z^{3}}+O\left(\frac{1}{z^{4}}\right) .
$$

Редукция при этом задается системой уравнений

$$
\begin{aligned}
& \frac{\partial p}{\partial u}=\frac{p^{2}-V p+R+u}{p^{3}-V p^{2}+R p+H} \\
& \frac{\partial p}{\partial v}=\frac{p-V}{p^{3}-V p^{2}+R p+H} \\
& \frac{\partial p}{\partial w}=\frac{1}{p^{3}-V p^{2}+R p+H}
\end{aligned}
$$

где многочлен $p^{3}-V p^{2}+R p+H$ имеет три простых корня. Условия совместности (13) при этом формулируются в виде

$$
\begin{array}{ll}
V_{v}=-R_{w}, & V_{u}=H_{w}+u V_{w} \\
R_{v}=-H_{w}+R V_{w}-V R_{w}, & R_{u}=V H_{w}-H V_{w}+u R_{w}-2 \\
H_{v}=1-V H_{w}+H V_{w}, & H_{u}=-V+R H_{w}-H R_{w}+u H_{w} .
\end{array}
$$


Выбирая теперь $S=S_{+}(p)$ и $t_{n}=0, n>4$, и принимая во внимание уравнения (15) и то, что $a_{1}=-b_{1}=-u$ и $a_{2}=-b_{2}=-v$, получим

$$
\frac{\partial S_{+}(p)}{\partial p}=4 t_{4}\left(p^{3}-V p^{2}+R p-H\right)=x+2 p y+3\left(p^{2}-u\right) t+4\left(p^{3}-2 u p-v\right) t_{4} .
$$

Из этого уравнения становится ясно, что решения для двух первых членов бездисперсионной иерархии КП могут быть получены, если решить систему

$$
V=-\frac{3 t}{4 t_{4}}, \quad R=\frac{y}{2 t_{4}}-2 u, \quad H=v-\frac{x-3 t u}{4 t_{4}} .
$$

Например, выбирая в (41) функцию $V$ в виде $V=V(u, v)$, получим решение для двух первых членов бездисперсионной иерархии КП, неявно задаваемое трансцендентным уравнением

$k_{1}^{3} x-2 k_{1}^{2} k_{2} y+3 k_{1} k_{2}^{2} t+4\left(k_{1}^{2} k_{3}+3 k_{1} k_{2}-k_{2}^{3}\right) t_{4}+\left(12 k_{1}^{2} k_{2} t_{4}-3 k_{1}^{3} t\right) u+4 k_{1}^{3} k_{5} t_{4} e^{k_{1} u}=0$

и

$$
v=-\frac{k_{3}}{k_{1}}-\frac{3 t}{4 k_{1} t_{4}}-\frac{k_{2}}{k_{1}} u
$$

В частном случае $k_{5}=0$ получим

$$
u\left(x, y, t, t_{4}\right)=\frac{k_{1}^{3} x-2 k_{1}^{2} k_{2} y+3 k_{1} k_{2}^{2} t+4\left(k_{1}^{2} k_{3}+3 k_{1} k_{2}-k_{2}^{3}\right) t_{4}}{3 k_{1}^{2}\left(k_{1} t-4 k_{2} t_{4}\right)} .
$$

Благодарности. Работа частично поддержана СICYТ (проект РВ98-0821).

\section{Список литературы}

[1] I. M. Krichever. Commun. Pure Appl. Math. 1992. V. 47. P. 437; Commun. Math. Phys. 1992. V. 143. P. 415.

[2] Y. Kodama, J. Gibbons. Phys. Lett. A. 1989. V. 135. P. 167.

[3] J. Gibbons, S. P. Tsarev. Phys. Lett. A. 1996. V. 211. P. 19; 1999. V. 258. P. 263.

[4] Y. Kodama. Progr. Theor. Phys. Suppl. 1988. V. 95. P. 184; Phys. Lett. A. 1988. V. 129. P. 223.

[5] T. Takasaky, T. Takebe. Int. J. Mod. Phys. A. 1992. V. 7. Suppl. 1B. P. 889; Rev. Math. Phys. 1995. V. 7. P. 743.

[6] B. A. Kupershmidt. J. Phys. A. 1990. V. 23. P. 871.

[7] V.V. Geogjaev. The quasiclassical limit of the inverse scattering problem method. In: Singular Limits of Dispersive Waves. NATO ASI Ser. B. V. 320. Eds. N. Ercolani et al. New York: Plenum, 1994. P. 53; Y. Kodama, J. Gibbons. Solving dispersionless Lax equations. Ibid. P. 61; Y. Kodama. Phys. Lett. A. 1990. V. 147. P. 477.

[8] L. Bianchi. Lezioni di Geometria Differenziale. Bologna: Zanichelli, 1924. 